\title{
The IL18 Promoter Polymorphism, rs1946518, Is Associated with the Risk of Periodontitis in Japanese Women: The Kyushu Okinawa Maternal and Child Health Study
}

\author{
Keiko Tanaka, ${ }^{1,2}$ Yoshihiro Miyake, ${ }^{1,2}$ Takashi Hanioka, ${ }^{3}$ Shinya Furukawa, ${ }^{1,2}$ \\ Nobuyuki Miyatake ${ }^{4}$ and Masashi Arakawa ${ }^{5}$ \\ ${ }^{1}$ Department of Epidemiology and Preventive Medicine, Ehime University Graduate School of Medicine, Toon, \\ Ehime, Japan \\ ${ }^{2}$ Epidemiology and Medical Statistics Unit, Translational Research Center, Ehime University Hospital, Toon, \\ Ehime, Japan \\ ${ }^{3}$ Department of Preventive and Public Health Dentistry, Fukuoka Dental College, Fukuoka, Fukuoka, Japan \\ ${ }^{4}$ Department of Hygiene, Faculty of Medicine, Kagawa University, Kita-gun, Kagawa, Japan \\ ${ }^{5}$ Health Tourism Research Fields, Graduate School of Tourism Sciences, University of the Ryukyus, Nakagami- \\ gun, Okinawa, Japan
}

\begin{abstract}
Interleukin-18 (IL-18) is a proinflammatory cytokine that plays an important role in periodontitis and its polymorphisms might modulate the individual susceptibility to periodontitis. Only a limited number of studies on the association between IL18 single-nucleotide polymorphisms (SNPs) and the risk of periodontitis have been realized, however. The aim of this case-control study among young post-partum Japanese women (18 to 45 years) was to determine the impact of SNPs, rs1946518 (-607 C/A) and rs187238 $(-137 \mathrm{G} / \mathrm{C})$, on periodontitis. The two SNPs may be located within a transcription factor-binding element, thereby influencing transcription from the IL18 promoter. Subjects were 131 cases who had at least one tooth with a probing pocket depth of $\geq 4.0 \mathrm{~mm}$ and 1,017 periodontally healthy controls. Probing pocket depth measurements were performed between 1 and 12 months post-partum. In this population, the A allele of rs1946518 and the C allele of rs187238 are more common. After adjustment for age, education, smoking, and use of an interdental brush, compared with subjects with the AA or AC genotype of SNP rs1946518, those with the CC genotype had a significantly reduced risk of periodontitis (adjusted odds ratio $=0.54,95 \%$ confidence interval $=0.29-0.97$ ). No significant association was observed between rs187238 and the risk of periodontitis. Our study did not reveal any evidence of interaction between the IL18 polymorphisms and smoking. Our findings indicate that the IL18 promoter SNP, rs1946518, is a potential risk factor of periodontitis among young Japanese women.
\end{abstract}

Keywords: case-control study; IL18; Japan; periodontal disease; polymorphism

Tohoku J. Exp. Med., 2017 November, 243 (3), 159-164. (C) 2017 Tohoku University Medical Press

\section{Introduction}

Periodontal disease is a chronic inflammatory disease of the teeth-supporting structures, such as alveolar bone, periodontal ligaments, and gingiva. Microbial plaque, which accumulates in the gingival crevice region and induces an inflammatory response, initiates periodontal disease. Like other common multifactorial diseases, genetic and environmental risk factors influence the development and progression of periodontal disease (Zhang et al. 2011; Genco and Borgnakke 2013). Genetic host factors may be implicated in determining susceptibility to periodontal disease (Zhang et al. 2011), which varies widely among indi- viduals (Genco and Borgnakke 2013).

Interleukin-18 (IL-18), a member of the IL-1 family of cytokines, is a potent proinflammatory cytokine that plays an important role in periodontal disease (Orozco et al. 2007). One of the main functions of IL-18 is to promote the production of interferon- $\gamma$ in the presence of IL-12 and to promote Th1 responses (Dinarello and Fantuzzi 2003). However, IL-18 also promotes Th2 responses. IL-18 promotes the secretion of other proinflammatory cytokines such as tumor necrosis factor- $\alpha$, IL- $1 \beta$, IL- 8 , and granulocyte-macrophage colony-stimulating factor, which enhances the expansion, migration, and activation of neutrophils during infections (Sahoo et al. 2011). The balance of the Th1/

Received August 18, 2017; revised and accepted October 19, 2017. Published online November 10, 2017; doi: 10.1620/tjem.243.159.

Correspondence: Keiko Tanaka, D.D.S., Ph.D., Department of Epidemiology and Preventive Medicine, Ehime University Graduate School of Medicine, Shitsukawa, Toon, Ehime 791-0295, Japan.

e-mail: tanaka.keiko.jn@ehime-u.ac.jp 
Th2 immune response contributes to initiation and progression of periodontal disease (Orozco et al. 2007). High levels of IL-18 are detected in inflammatory and autoimmune diseases such as rheumatoid arthritis, psoriasis, and systemic lupus erythematosus (Mühl and Pfeilschifter 2004). Increasing evidence indicated that IL-18 has the property of inhibiting bone resorption; therefore, IL-18 would be expected to contribute to the regulation of bone in inflammatory bone diseases, including periodontal disease (Silva et al. 2015). Thus, IL-18 might affect periodontal health and disease through various functions including the production, signaling pathways, and the inhibition of bone resorption (Orozco et al. 2007).

IL-18 protein expression is regulated by the ILI8 promoter gene. Several polymorphisms in the promoter region of IL18 have been identified (Giedraitis et al. 2001). The two single-nucleotide polymorphisms (SNPs) at positions -607 and -137 have been extensively studied in relation to multifactorial diseases such as asthma, systemic lupus erythematosus, cardiovascular disease, and diabetes. Each of these two SNPs may be located within a transcription factor-binding element, thereby influencing transcription from the IL18 promoter gene (Giedraitis et al. 2001; Thompson and Humphries 2007). To our knowledge, only four studies have examined the association between IL18 polymorphism at positions -607 and -137 and periodontal disease; these were conducted in Italian adults $>50$ years old $(30 \%$ male patients) (Martelli et al. 2012) and in German subjects with mean ages of 52.9 years (54\% male patients) (Folwaczny et al. 2005), 34.0 years ( $42 \%$ male patients) (Noack et al. 2008), and 57.3 years (44\% male patients) (Noack et al. 2009) and yielded inconsistent results. According, however, to a meta-analysis based on only three studies in Germany, IL18 polymorphism was associated with an increased risk of periodontal disease ( $\mathrm{Li}$ et al. 2014). It is necessary to accumulate further evidence in order to clarify how IL18 polymorphisms affect periodontal disease.

In the present study, we assessed associations between two IL18 SNPs, namely, rs1946518 (-607 C/A) and rs187238 $(-137 \mathrm{G} / \mathrm{C})$, and the risk of periodontal disease among young Japanese women, using the data set of the Kyushu Okinawa Maternal and Child Health Study (KOMCHS). Furthermore, haplotype analyses were performed. As smoking is an established risk factor for periodontal disease, we investigated the possibility of interactions between the SNPs and smoking.

\section{Materials and methods}

Study population

The KOMCHS is an ongoing prospective prebirth cohort study conducted in seven prefectures on Kyushu Island in southern Japan or Okinawa Prefecture. Details of the KOMCHS have been described previously (Miyake et al. 2013; Tanaka et al. 2013). In brief, at 423 obstetric hospitals, pregnant women were recruited between April 2007 and March 2008. Finally, a total of 1,757 pregnant women between the 5th and 39th week of pregnancy gave their fully informed written consent to participate and completed the baseline survey, which included questions regarding on smoking habits, education, and use of an interdental brush. Of these, 1,590 mothers completed the second survey after birth, and of these, 1,198 women received oral examinations between 1 and 12 months post-partum. Around four months after delivery, 1,492 mothers gave informed consent for genotyping. The present study subjects consisted of 1,157 women who both received oral examinations and provided genetic samples. The ethics committees of the Faculty of Medicine, Fukuoka University and Ehime University Graduate School of Medicine approved the KOMCHS.

\section{Selection of cases and control subjects}

Dental hygienists performed oral examinations for periodontal status. Probing pocket depth (PPD) was assessed with a Community Periodontal Index probe (YDM Corp., Tokyo, Japan) at six sites per tooth for six teeth: the right first molar, right first incisor, and left first molar in the maxilla and the right first molar, left first incisor, and left first molar in the mandible. Periodontal disease was defined as a case where there is a PPD of $4.0 \mathrm{~mm}$ or more at any site. Under this definition, among the 1,157 subjects, 131 women had periodontal disease. The remaining subjects were eligible to be used as controls, but nine women were excluded due to missing data on the variables under study; thus 1,017 women were classified as control subjects.

\section{DNA extraction and genotyping}

Buccal specimens were collected with BuccalAmp swabs (Epicenter BioTechnologies, Madison, WI, USA). Genomic DNA was extracted using a QIAmp DNA mini kit (Qiagen, Inc., Valencia, CA, USA). Genotyping of IL18 SNPs was performed using TaqMan SNP Genotyping Assays on a StepOnePlus machine (Applied Biosystems, Foster City, CA, USA) according to the manufacturer's instructions. Genotypes were determined by TaqMan probes assay ID C_2898460_10 and C_2408543_10 for rs1946518 and rs 187238, respectively.

\section{Statistical analysis}

The chi-square test was used to test for departures from the Hardy-Weinberg equilibrium among the control subjects. Linkage disequilibrium was examined using Haploview software version 4.2 (Broad Institute, Cambridge, MA, USA) (Barrett et al. 2005). To test for genetic associations, logistic regression was used to compute crude odds ratios (ORs) and 95\% confidence intervals (CIs), with the reference category being the homozygote of the major allele. The following variables were adjusted in the multivariable model: age at oral examination, education, smoking, and use of an interdental brush. The statistical power was calculated using QUANTO version 1.2 (Gauderman 2002). Haplotypes and their frequencies were inferred according to the expectation maximization algorithm. We estimated whether haplotype frequencies differ between the cases and control groups, using crude ORs and 95\% CIs that were based on the frequency of each haplotype relative to all other haplotypes combined. We assessed multiplicative interactions between the SNPs under study and smoking with regard to the risk of periodontal disease, using a multiplicative term included in a multivariate model. Excluding the calculation of linkage disequilibrium and statistical power, all statistical analyses were performed using STATA/SE software version 12.0 (StataCorp, College Station, TX, USA). 


\section{Results}

The demographic characteristics of study subjects are summarized in Table 1. There were no differences in distributions of age, education, smoking status, or use of an interdental brush between cases and controls.

In the controls, the genotype distributions of IL 18 SNPs rs 1946518 and rs187238 were consistent with HardyWeinberg equilibrium ( $P=0.75$ and 0.55 , respectively). The two SNPs under study were in tight linkage disequilibrium $\left(D^{\prime}=1, r^{2}=0.09\right)$.
No evident relationships were observed between SNP rs1946518 and periodontal disease under the co-dominant or dominant model (Table 2). In the recessive model, compared with subjects with the AA or AC genotype of SNP rs1946518, those with the CC genotype had a significantly reduced risk of periodontal disease (adjusted OR $=0.54$, $95 \% \mathrm{CI}=0.29-0.97$ ). No evident association was observed between rs 187238 and the risk of periodontal disease under any genetic models. With regard to SNP rs 187238 , the statistical power calculation revealed that, using our sample size, we could detect the gene-disease association for an

Table 1. Characteristics of the study population.

\begin{tabular}{lcc}
\hline & \multicolumn{2}{c}{$n(\%)$} \\
\cline { 2 - 3 } Variable & Cases & Controls \\
\hline Age, years, mean \pm SD & $(n=131)$ & $(n=1,017)$ \\
(median, range) & $32.9 \pm 4.1$ & $32.3 \pm 4.2$ \\
Education, years & $(33,21-41)$ & $(32,18-45)$ \\
$<13$ & & \\
$13-14$ & $23(17.6)$ & $210(20.7)$ \\
$\quad \geq 15$ & $50(38.2)$ & $337(33.1)$ \\
Smoking & $58(44.3)$ & $470(46.2)$ \\
$\quad$ Never & & \\
Ever & $86(65.7)$ & $722(71.0)$ \\
Use of an interdental brush & $45(34.4)$ & $295(29.0)$ \\
No & & \\
Yes & $64(48.9)$ & $545(53.6)$ \\
\hline
\end{tabular}

Table 2. ORs and $95 \%$ CIs for periodontal disease associated with IL18 polymorphisms in young Japanese women.

\begin{tabular}{|c|c|c|c|c|c|c|}
\hline \multirow[t]{2}{*}{ SNP } & \multirow[t]{2}{*}{ Genotype } & \multicolumn{2}{|c|}{ Number $(\%)$ of } & \multirow[t]{2}{*}{ Crude OR $(95 \% \mathrm{CI})$} & \multirow[t]{2}{*}{ Adjusted OR $(95 \% \mathrm{CI})^{*}$} & \multirow[t]{2}{*}{$P$ value } \\
\hline & & Cases $(n=131)$ & Controls $(n=1,017)$ & & & \\
\hline \multirow[t]{5}{*}{ rs 1946518} & $\mathrm{AA}$ & $46(35.1)$ & $344(33.8)$ & 1.00 & 1.00 & \\
\hline & $\mathrm{AC}$ & $72(55.0)$ & $500(49.2)$ & $1.08(0.73-1.60)$ & $1.07(0.72-1.58)$ & 0.76 \\
\hline & $\mathrm{CC}$ & $13(9.9)$ & $173(17.0)$ & $0.56(0.30-1.07)$ & $0.56(0.29-1.06)$ & 0.08 \\
\hline & $\mathrm{AC}+\mathrm{CC}$ vs. AA & & & $0.94(0.64-1.38)$ & $0.93(0.64-1.37)$ & 0.73 \\
\hline & $\mathrm{CC}$ vs. $\mathrm{AA}+\mathrm{AC}$ & & & $0.54(0.30-0.98)$ & $0.54(0.29-0.97)$ & 0.04 \\
\hline \multirow[t]{5}{*}{ rs 187238} & $\mathrm{CC}$ & $108(82.4)$ & $793(78.0)$ & 1.00 & 1.00 & \\
\hline & CG & $21(16.0)$ & $208(20.5)$ & $0.74(0.45-1.21)$ & $0.73(0.45-1.20)$ & 0.22 \\
\hline & GG & $2(1.5)$ & $16(1.6)$ & $0.92(0.21-4.05)$ & $0.86(0.19-3.83)$ & 0.85 \\
\hline & $\mathrm{CG}+\mathrm{GG}$ vs. $\mathrm{CC}$ & & & $0.75(0.47-1.21)$ & $0.74(0.46-1.20)$ & 0.22 \\
\hline & GG vs. $\mathrm{CC}+\mathrm{CG}$ & & & $0.97(0.22-4.27)$ & $0.91(0.21-4.04)$ & 0.90 \\
\hline
\end{tabular}

*Adjusted for age at oral examination, education, smoking, and use of an interdental brush.

CI, confidence interval; OR, odds ratio; SNP, single-nucleotide polymorphism. 
Table 3. Haplotype analysis of 2 IL 18 polymorphisms associated with periodontal disease in young Japanese women*.

\begin{tabular}{|c|c|c|c|c|}
\hline \multirow[b]{2}{*}{ Haplotype $^{\dagger}$} & \multicolumn{2}{|l|}{ Frequency, $n(\%)$} & \multirow[b]{2}{*}{ Crude OR $(95 \% \mathrm{CI})^{*}$} & \multirow[b]{2}{*}{$P$ value } \\
\hline & Cases $(2 n=262)$ & Controls $(2 n=2,034)$ & & \\
\hline $\mathrm{AC}$ & $139(53.1)$ & $948(46.6)$ & $1.29(0.99-1.69)$ & 0.05 \\
\hline $\mathrm{CC}$ & $98(37.4)$ & $846(41.6)$ & $0.84(0.64-1.10)$ & 0.19 \\
\hline $\mathrm{AG}$ & $25(9.5)$ & $240(11.9)$ & $0.79(0.49-1.22)$ & 0.28 \\
\hline
\end{tabular}

*Rare haplotypes (frequencies less than $1 \%$ in either cases or controls) were deleted.

"Haplotype order is rs 1946518 and rs 187238.

${ }^{\ddagger}$ Crude OR for each haplotype is relative to all other haplotypes combined.

CI, confidence interval; OR, odds ratio; SNP, single-nucleotide polymorphism.

Table 4. Association between IL18 polymorphisms and periodontal disease, stratified by smoking history in young Japanese women.

\begin{tabular}{|c|c|c|c|c|c|c|c|c|}
\hline \multirow[t]{3}{*}{ SNP } & \multirow[t]{3}{*}{ Genotype } & \multicolumn{6}{|l|}{ Smoking history } & \multirow[t]{3}{*}{$P$ for interaction } \\
\hline & & \multicolumn{3}{|l|}{ No $(n=808)$} & \multicolumn{3}{|l|}{ Yes $(n=340)$} & \\
\hline & & No. cases/controls & $\begin{array}{l}\text { Adjusted OR } \\
(95 \% \mathrm{CI})^{*}\end{array}$ & $P$ value & No. cases/controls & $\begin{array}{l}\text { Adjusted OR } \\
(95 \% \mathrm{CI})^{*}\end{array}$ & $P$ value & \\
\hline \multirow[t]{2}{*}{ rs1946518 } & $\mathrm{AA}+\mathrm{AC}$ & $76 / 594$ & 1.00 & & $42 / 250$ & 1.00 & & \\
\hline & $\mathrm{CC}$ & $10 / 128$ & $0.60(0.30-1.19)$ & 0.15 & $3 / 45$ & $0.37(0.11-1.25)$ & 0.11 & 0.16 \\
\hline
\end{tabular}

*Adjusted for age at oral examination, education, and use of an interdental brush.

CI, confidence interval; OR, odds ratio; SNP, single-nucleotide polymorphism.

OR of 0.495 with an accuracy of more than $80 \%$ at a significance level of 0.05 with a two-sided alternative hypothesis under the dominant model.

Given the haplotype order of rs 1946518 and rs 187238 , AC, CC, and AG haplotypes were identified after haplotypes with a frequency of $<1 \%$ in either cases or controls had been deleted. None of these were significantly related to the risk of periodontal disease, however (Table 3).

We observed no significant interaction between SNP rs1946518 and smoking in terms of the risk of periodontal disease (Table 4).

\section{Discussion}

To our knowledge, this is the first study in a non-Western population to examine the association between IL18 polymorphisms and periodontal disease. The present study showed that the CC genotype of IL18 SNP rs1946518 was significantly associated with a reduced risk of the disease. There was no association between IL18 SNP rs187238 and periodontal disease. A case-control study in Italy (109 aggressive cases, 104 chronic cases, and 100 controls) showed that the AA genotype of SNP rs 1946518 was positively associated with chronic periodontal disease, while the CG genotype of SNP rs187238 was positively associated with aggressive periodontal disease (Martelli et al. 2012). In three other previous studies in Germany, no significant association was observed between IL18 polymorphisms and chronic periodontal disease (108 cases and 76 controls in one study, 123 cases and 121 controls in another)
(Folwaczny et al. 2005; Noack et al. 2009) or aggressive periodontal disease (111 cases and 80 controls) (Noack et al. 2008). It should be noted, however, that these previous studies used different definitions of periodontal disease, study populations, and confounding factors, thus limiting the feasibility of inter-study comparisons. There are also several genome-wide association studies on periodontal disease (Teumer et al. 2013; Shaffer et al. 2014; Shimizu et al. 2015), but none of these has reported an association for IL18 SNP. This is likely to be attributed to the low power of the studied sample sizes to reach genome-wide significance.

The frequency distribution of genetic variants is different between populations. In our study population, the A allele frequency of rs 1946518 was $58.9 \%$ and the $\mathrm{C}$ allele frequency of rs 187238 was $88.5 \%$. On the other hand, previous studies in Western populations such as in Italy and Germany demonstrated that A allele frequency of rs 1946518 and $\mathrm{C}$ allele frequency of rs 187238 ranged from $38.5 \%$ to $40.7 \%$ and from $24.8 \%$ to $27.7 \%$, respectively (Folwaczny et al. 2005; Noack et al. 2008, 2009; Martelli et al. 2012).

IL-18 contributes to both host defense and inflammation in a cascade of cytokines associated with innate responses. A case-control study in Mexico (18 cases and 9 controls) demonstrated that serum IL-18 levels were higher in subjects with chronic periodontal disease than in controls (Sánchez-Hernández et al. 2011). In a case control study in Turkey (22 cases and 21 controls), salivary levels of IL-18 were significantly higher in patients with chronic periodon- 
tal disease than in healthy control subjects, although there was no difference in plasma IL-18 levels (Ozçaka et al. 2011). A meta-analysis based on six studies suggested that higher levels of plasma IL-18 were associated with periodontal disease, especially among Asian populations ( $\mathrm{Li}$ et al. 2014).

The two SNPs rs1946518 and rs187238 may influence the gene expression of IL18 at the transcriptional level by disrupting the cyclic adenosine monophosphate-responsive element binding site and by changing the H4TF-1 nuclear factor binding at the IL18 promoter (Giedraitis et al. 2001). However, it is still unclear whether IL18 polymorphisms are implicated in inter-individual serum IL-18 level variation (Thompson and Humphries 2007). Recently, a casecontrol study of bladder cancer in Northern India reported that the heterozygous variant genotype of IL18 rs 187238 was significantly associated with elevated IL-18 serum level (Jaiswal et al. 2013).

The methodological strengths in the study were as follows: study subjects were homogeneous in gender and age group and we were able to control for several confounding factors.

We also recognized certain limitations in the present study, however. First, the rate of participation cannot be calculated due to lack of information for the exact number of eligible pregnant women who were recruited at obstetric hospitals. Additionally, it was impossible to assess differences of personal characteristics between participants and non-participants, because demographic data were not available. It is unlikely that our subjects were a representative sample of Japanese women in the general population. In fact, our study subjects have higher educational levels than do those in the general population (Statistics Bureau, Ministry of Public Management, Home Affairs, Posts and Telecommunications 2002). Additionally, according to the Survey of Dental Disease conducted in 2011 (Japanese Society for Dental Health 2013), the proportion of women aged 30 to 34 years having one or more periodontal sites with a PPD of $4.0 \mathrm{~mm}$ or deeper was $14.2 \%$. The corresponding figure for our subjects was $11.4 \%$. It was expected that our study population would have had a greater awareness and knowledge of health than the general population has. However, the distribution of both SNPs investigated was consistent with the Hardy-Weinberg equilibrium.

Second, in the current study, dental hygienists performed oral examinations. The hygienists were given detailed criteria for carrying out the examinations, but in order to standardize the procedure no specific training was provided. Further, no reliability assessment of measurements was carried out in the present study. Thus, it is uncertain whether intra- and inter-examiner consistency was established. Further, because data on partial-mouth examinations was used, the periodontal disease prevalence is likely to be underestimated. In the present study, no radiographic evaluation of alveolar bone loss was made. In addition, PPD measurements were performed between 1 and 12 months post-partum. Therefore, we could not exclude the possibility that periodontal changes such as increasing PPD depth and bleeding on probing or mechanical stimulation during pregnancy might remain. Thus, we need to be aware of the possibility of false positive errors in our outcome.

Third, although adjustment was made for some confounders, residual confounding cannot be ruled out.

Fourth, we did not confirm the Japanese ancestry of the study participants. Because admixture is a well-known confounder in genetic association studies, observed associations in the present study might be related to ancestry rather than disease.

Within the limits of the present study, our results demonstrate that the CC genotype of SNP rs1946518 was significantly associated with a reduced risk of periodontal disease in young post-partum Japanese women. None of the ILI8 haplotypes tested in the present study were associated with increased risk of periodontal disease, and there was no interaction of any of the IL18 polymorphisms with smoking. Additional research is required to confirm these findings and to identify the mechanisms behind the observed association.

\section{Acknowledgments}

This study was supported by Japan Society for the Promotion of Science KAKENHI grants (JP19590606, JP20791654, JP21590673, JP22592355, JP22119507, JP24390158, JP25463275, JP25670305 and JP17K12011); Health and Labour Sciences Research Grants, Research on Allergic Disease and Immunology from the Ministry of Health, Labour, and Welfare of Japan; and the Takeda Science Foundation.

The authors would like to acknowledge the Kyushu Branch of the Japan Allergy Foundation, the Fukuoka Association of Obstetricians \& Gynecologists, the Okinawa Association of Obstetricians \& Gynecologists, the Miyazaki Association of Obstetricians \& Gynecologists, the Oita Association of Obstetricians \& Gynecologists, the Kumamoto Association of Obstetricians \& Gynecologists, the Nagasaki Association of Obstetricians \& Gynecologists, the Kagoshima Association of Obstetricians \& Gynecologists, the Saga Association of Obstetricians \& Gynecologists, the Fukuoka Society of Obstetrics and Gynecology, the Okinawa Society of Obstetrics and Gynecology, the Fukuoka Dental Hygienists' Association, the Okinawa Dental Hygienists' Association, the Miyazaki Dental Hygienists' Association, the Oita Dental Hygienists' Association, the Kumamoto Dental Hygienists' Association, the Nagasaki Dental Hygienists' Association, the Kagoshima Dental Hygienists' Association, the Saga Dental Hygienists' Association, the Fukuoka City Government, and the Fukuoka City Medical Association for their valuable support, as well as Mrs. Yukari Hayashi for her technical assistance.

\section{Conflict of Interest}

The authors declare no conflict of interest.

\section{References}

Barrett, J.C., Fry, B., Maller, J. \& Daly, M.J. (2005) Haploview: analysis and visualization of LD and haplotype maps. Bioinformatics, 21, 263-265.

Dinarello, C.A. \& Fantuzzi, G. (2003) Interleukin-18 and host 
defense against infection. J. Infect. Dis., 187, S370-384.

Folwaczny, M., Glas, J., Török, H.P., Tonenchi, L., Paschos, E., Bauer, B., Limbersky, O. \& Folwaczny, C. (2005) Polymorphisms of the interleukin-18 gene in periodontitis patients. $J$. Clin. Periodontol., 32, 530-534.

Gauderman, W.J. (2002) Sample size requirements for matched case-control studies of gene-environment interaction. Stat. Med., 21, 35-50.

Genco, R.J. \& Borgnakke, W.S. (2013) Risk factors for periodontal disease. Periodontol. 2000, 62, 59-94.

Giedraitis, V., He, B., Huang, W.X. \& Hillert, J. (2001) Cloning and mutation analysis of the human IL-18 promoter: a possible role of polymorphisms in expression regulation. J. Neuroimmunol., 112, 146-152.

Jaiswal, P.K., Singh, V., Srivastava, P. \& Mittal, R.D. (2013) Association of IL-12, IL-18 variants and serum IL-18 with bladder cancer susceptibility in North Indian population. Gene, 519, $128-134$.

Japanese Society for Dental Health (2013) Report on the Survey of Dental Diseases 2011. Tokyo: Japanese Society for Oral Health.

Li, Z.G., Li, J.J., Sun, C.A., Jin, Y. \& Wu, W.W. (2014) Interleukin-18 promoter polymorphisms and plasma levels are associated with increased risk of periodontitis: a meta-analysis. Inflamm. Res., 63, 45-52.

Martelli, F.S., Mengoni, A., Martelli, M., Rosati, C. \& Fanti, E. (2012) IL-18 gene promoter polymorphisms are only moderately associated with periodontal disease in Italian population. Clin. Cases Miner. Bone Metab., 9, 153-156.

Miyake, Y., Tanaka, K., Okubo, H., Sasaki, S. \& Arakawa, M. (2013) Fish and fat intake and prevalence of depressive symptoms during pregnancy in Japan: baseline data from the Kyushu Okinawa Maternal and Child Health Study. $J$. Psychiatr. Res., 47, 572-578.

Mühl, H. \& Pfeilschifter, J. (2004) Interleukin-18 bioactivity: a novel target for immunopharmacological anti-inflammatory intervention. Eur. J. Pharmacol., 500, 63-71.

Noack, B., Görgens, H., Lorenz, K., Schackert, H.K. \& Hoffmann, T. (2009) TLR4 and $I L-18$ gene variants in chronic periodontitis: impact on disease susceptibility and severity. Immunol. Invest., 38, 297-310.

Noack, B., Görgens, H., Lorenz, K., Ziegler, A., Hoffmann, T. \& Schackert, H.K. (2008) TLR4 and IL-18 gene variants in aggressive periodontitis. J. Clin. Periodontol., 35, 1020-1026.

Orozco, A., Gemmell, E., Bickel, M. \& Seymour, G.J. (2007) Interleukin 18 and periodontal disease. J. Dent. Res., 86, 586-593.

Ozçaka, O., Nalbantsoy, A. \& Buduneli, N. (2011) Interleukin-17 and interleukin-18 levels in saliva and plasma of patients with chronic periodontitis. J. Periodontal Res., 46, 592-598.

Sahoo, M., Ceballos-Olvera, I., del Barrio, L. \& Re, F. (2011) Role of the inflammasome, IL- $\beta$, and IL-18 in bacterial infections. Scientific World Journal, 11, 2037-2050.

Sánchez-Hernández, P.E., Zamora-Perez, A.L., Fuentes-Lerma, M., Robles-Gómez, C., Mariaud-Schmidt, R.P. \& GuerreroVelázquez, C. (2011) IL-12 and IL-18 levels in serum and gingival tissue in aggressive and chronic periodontitis. Oral Dis., 17, 522-529.

Shaffer, J.R., Polk, D.E., Wang, X., Feingold, E., Weeks, D.E., Lee, M.K., Cuenco, K.T., Weyant, R.J., Crout, R.J., McNeil, D.W. \& Marazita, M.L. (2014) Genome-wide association study of periodontal health measured by probing depth in adults ages 18-49 years. G3 (Bethesda), 4, 307-314.

Shimizu, S., Momozawa, Y., Takahashi, A., Nagasawa, T., Ashikawa, K., Terada, Y., Izumi, Y., Kobayashi, H., Tsuji, M., Kubo, M. \& Furuichi, Y. (2015) A genome-wide association study of periodontitis in a Japanese population. J. Dent. Res., 94, 555-561.

Silva, N., Abusleme, L., Bravo, D., Dutzan, N., Garcia-Sesnich, J., Vernal, R., Hernández, M. \& Gamonal, J. (2015) Host response mechanisms in periodontal diseases. J. Appl. Oral Sci., 23, 329-355.

Statistics Bureau, Ministry of Public Management, Home Affairs, Posts and Telecommunications (2002) 2000 Population Census of Japan, Vol. 3-2-40, Labour Force Status of Population, Industry (Major Groups) of Employed Persons, and Education, Fukuoka-ken. Tokyo: Statistics Bureau, Ministry of Public Management, Home Affairs, Posts and Telecommunications, p. 666.

Tanaka, K., Miyake, Y., Hanioka, T. \& Arakawa, M. (2013) VDR gene polymorphisms, interaction with smoking and risk of periodontal disease in Japanese women: the Kyushu Okinawa maternal and child health study. Scand. J. Immunol., 78, 371-377.

Teumer, A., Holtfreter, B., Völker, U., Petersmann, A., Nauck, M., Biffar, R., Völzke, H., Kroemer, H.K., Meisel, P., Homuth, G. \& Kocher, T. (2013) Genome-wide association study of chronic periodontitis in a general German population. J. Clin. Periodontol., 40, 977-985.

Thompson, S.R. \& Humphries, S.E. (2007) Interleukin-18 genetics and inflammatory disease susceptibility. Genes Immun., 8, 91-99.

Zhang, J., Sun, X., Xiao, L., Xie, C., Xuan, D. \& Luo, G. (2011) Gene polymorphisms and periodontitis. Periodontol. 2000, 56, 102-124. 